\title{
Residual stress preserved in quartz from the San Andreas Fault Observatory at Depth.
}

Kai Chen, Martin Kunz, Nobumichi Tamura and Hans-Rudolf Wenk

\section{Supplementary Material}

\section{Section 1. Type and origin of residual stresses}

For the purpose of this paper, we are interested in what are known as intragranular microstresses, i.e. stresses on the length scale of individual grains. Their origin is related to the microstructural evolution of a non-uniform or anisotropic polycrystalline solid, due to factors such as the anisotropy of the elastic modulus and/or yield strength, and/or the mismatch of thermal expansion coefficients between adjacent grains undergoing temperature fluctuations. Residual microstresses at the intragranular scale can also result from the elastic strain field associated with impurities such as vacancies, substitutional or interstitial atoms, dislocations and disclinations, or by intergranular interactions in a heterogeneous polyphase aggregate.

Although a macroscopic deformation is usually controlled by the amount and distribution of microscopic strain, in the case of heterogeneous polycrystalline and/or multiphase solids, the difference between macrostresses and local microstresses can be significant (Sih and Tang, 2006; Wawszczak et al., 2011; Withers and Bhadeshia, 2001). In order to fully understand and precisely describe the elastic stress and elastic energy distribution in such a complex system, we need to be able to spatially resolve strain/stress distributions at a sub-grain level. Synchrotron X-ray microdiffraction ( $\mu$ XRD) is a technique which is able to map the stress distribution at the grain scale at different locations on a thin section. In Section 3 we will demonstrate 5 scans, taken from a variety of quartz grains, in particular a synthetic quartz crystal which we use as a reference, and 4 scans of quartz inside a SAFOD fault cataclasite.

\section{Section 2. Measurement of strain/stress tensors}

To measure and express the orientation and strain/stress tensors of a crystal grain, three sets of coordinate systems are required, including the laboratory coordinates $\boldsymbol{O}-\boldsymbol{x y z}$, the lattice coordinates $\boldsymbol{O}-\boldsymbol{a b} \boldsymbol{c}$, and the orthonormal lattice coordinates $\boldsymbol{O}-\boldsymbol{a}^{\boldsymbol{o}} \boldsymbol{b}^{\boldsymbol{o}} \boldsymbol{c}^{\boldsymbol{o}}$.

The laboratory coordinates are in most cases established as a Cartesian coordinate system. In this study, we define the $z$-axis normal to the thin section specimen surface, while $\boldsymbol{x}$ - and $\boldsymbol{y}$-axes are parallel with the horizontal and vertical scanning directions, respectively.

The lattice coordinate system is built up upon the three basic vectors of the crystal lattice $(\boldsymbol{a}, \boldsymbol{b}$, and $\boldsymbol{c}$ ), and thus it is not necessarily Cartesian, depending on the symmetry of the crystal structure. The orientation of a crystal grain is readily represented by a 
transformation matrix $\boldsymbol{M}_{\boldsymbol{u}}$ between the lattice coordinates $\boldsymbol{O}-\boldsymbol{x y} z$ and the laboratory coordinates $\boldsymbol{O}-\boldsymbol{a b c}$

$$
\boldsymbol{M}_{\boldsymbol{u}}=\left[\begin{array}{lll}
a_{x, u} & a_{y, u} & a_{z, u} \\
b_{x, u} & b_{y, u} & b_{z, u} \\
c_{x, u} & c_{y, u} & c_{z, u}
\end{array}\right]
$$

where $\boldsymbol{a}, \boldsymbol{b}, \boldsymbol{c}$, and $\boldsymbol{x}, \boldsymbol{y}, \boldsymbol{z}$ are the unit vectors of $\boldsymbol{O}-\boldsymbol{a b} \boldsymbol{c}$ and $\boldsymbol{O}-\boldsymbol{x y} \boldsymbol{z}$, respectively. The subscript $u$ refers to the unstrained lattice. For the sake of simplicity, $\boldsymbol{M}_{\boldsymbol{u}}$ is re-written to be $\boldsymbol{M u}=\left[\boldsymbol{a}_{\boldsymbol{u}} \boldsymbol{b}_{\boldsymbol{u}} \boldsymbol{c}_{\boldsymbol{u}}\right]^{\mathrm{T}}$, where $\boldsymbol{a}_{\boldsymbol{u}}, \boldsymbol{b}_{\boldsymbol{u}}$, and $\boldsymbol{c}_{\boldsymbol{u}}$ are column vectors and the superscript T means transpose. In practice, $\boldsymbol{M u}$ is obtained by indexing a Laue pattern from a single crystal, i.e. fitting the Laue reflection peak positions measured experimentally with the ones calculated based on the unstrained lattice parameters.

Once a Laue diffraction is indexed, the deviatoric strain refinement is carried out by varying the ratio of $c / a, b / a$ as well as the three lattice parameter angles $\alpha, \beta$, and $\gamma$, and re-calculating the diffraction peak positions accordingly, until the deviation between the calculated peak positions and the measured ones is minimized. The refined lattice is then defined by three basic vectors $\boldsymbol{a}_{\boldsymbol{r}}, \boldsymbol{b}_{\boldsymbol{r}}$, and $\boldsymbol{c}_{\boldsymbol{r}}$ and the refined orientation matrix is

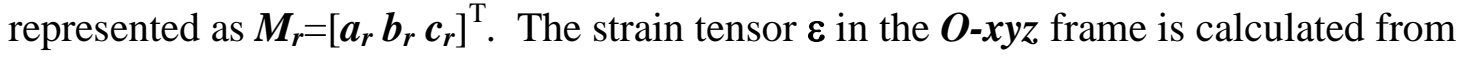
the following equation:

$$
\varepsilon_{x y z}=\frac{1}{2}\left(\boldsymbol{I}-\boldsymbol{M}_{r}^{-1} \cdot \boldsymbol{M}_{u} \cdot \boldsymbol{M}_{u}^{T} \cdot\left(\boldsymbol{M}_{r}^{-1}\right)^{T}\right)
$$

where $I$ is the $3 \times 3$ unity matrix, and the superscript -1 denotes inverse (Pavese, 2005). Since the Laue technique is insensitive to the lattice volume change, only the deviatoric strain tensor $\boldsymbol{\varepsilon}^{\prime}$ can be measured, which is linked to the full strain tensor $\boldsymbol{\varepsilon}$ by the following relationship:

$$
\varepsilon_{x y z}^{\prime}=\varepsilon_{x y z}-\frac{\operatorname{tr}\left(\varepsilon_{x y z}\right)}{3}
$$

where $\operatorname{tr}$ means matrix trace.

In order to calculate the stress tensor, an orthonormal coordinate system $\boldsymbol{O}-\boldsymbol{a}^{\boldsymbol{o}} \boldsymbol{b}^{\boldsymbol{o}} \boldsymbol{c}^{\boldsymbol{o}}$ is defined, in which the stiffness constants $\left(\boldsymbol{C}_{i j}\right)$ are usually reported in the literature. $\boldsymbol{O}$ $\boldsymbol{a}^{\boldsymbol{o}} \boldsymbol{b}^{\boldsymbol{o}} \boldsymbol{c}^{\boldsymbol{o}}$ is defined based on $\boldsymbol{O}-\boldsymbol{a b c}$, and in this study we follow the definition suggested by Matthies et al. (1988), in which the $\boldsymbol{c}^{\boldsymbol{o}}$-axis is collinear with the $\boldsymbol{c}$-axis, $\boldsymbol{b}^{\boldsymbol{o}}$ is perpendicular to the $\boldsymbol{a c}$-plane, $\boldsymbol{a}^{\boldsymbol{o}}$ lies hence in the $\boldsymbol{a c}$-plane to fulfill the right-handed criterion, and $\boldsymbol{a}^{\mathbf{o}}$, $\boldsymbol{b}^{\mathbf{o}}$ and $\boldsymbol{c}^{\mathbf{0}}$ are unit vectors. Therefore, the transformation matrix $\mathbf{L}$ that brings $\boldsymbol{O}-\boldsymbol{a}^{\boldsymbol{o}} \boldsymbol{b}^{\boldsymbol{o}} \boldsymbol{c}^{\boldsymbol{o}}$ to $\boldsymbol{O}-\boldsymbol{a b c}$ has the following form:

$$
\mathbf{L}=\left[\begin{array}{ccc}
a \cdot \sin \beta & -b \cdot \sin \alpha \cdot \cos \gamma^{*} & 0 \\
0 & b \cdot \sin \alpha \cdot \sin \gamma^{*} & 0 \\
a \cdot \cos \beta & b \cdot \cos \alpha & c
\end{array}\right]
$$

where $a, b, c, \alpha, \beta$, and $\gamma$ are the lattice parameters and $\gamma^{*}$ is the angle between $c^{*}$-axis and $\boldsymbol{a}^{*}$-axis in reciprocal lattice. Consequently, any column vector $\boldsymbol{v}$ (represented by three numbers) in lattice coordinates is equivalent to $v^{\boldsymbol{o}}$ in the orthonormal coordinates as long as they obey the following relationship: 


$$
v^{\mathbf{o}}=\mathbf{L} \cdot v \text { or } v=\mathbf{L}^{-1} \cdot v^{\mathbf{o}}
$$

The product $\boldsymbol{R}$ of $\boldsymbol{M}_{\boldsymbol{u}}$ and $\boldsymbol{L}^{-1}$ gives a pure rotation matrix, i.e. $\operatorname{det}(\boldsymbol{R})=1$. Thus any $2^{\text {nd }}$ rank tensor can be readily transformed between the $\boldsymbol{O}-\boldsymbol{a}^{\boldsymbol{o}} \boldsymbol{b}^{\boldsymbol{o}} \boldsymbol{c}^{\boldsymbol{o}}$ frame $\left(\delta_{a^{o} b^{o} c^{o}}\right)$ and the $\boldsymbol{O}-\boldsymbol{x y z}$ frame $\left(\delta_{x y z}\right)$ by applying the rotation:

$$
\delta_{a b c}=\boldsymbol{R}^{T} \delta_{x y z} \boldsymbol{R} \text { or } \quad \delta_{x y z}=R \delta_{a b c} R^{T}
$$

In most cases, the stiffness tensors $\boldsymbol{C}_{\mathbf{i j}}$ published in the literature are written in 6 $\times 6$ matrices form in the $\boldsymbol{O}-\boldsymbol{a}^{\boldsymbol{o}} \boldsymbol{b}^{\boldsymbol{o}} \boldsymbol{c}^{\boldsymbol{o}}$ frame, and thus the six stress tensor components, also in the $\boldsymbol{O}-\boldsymbol{a}^{\boldsymbol{o}} \boldsymbol{b}^{\boldsymbol{o}} \boldsymbol{c}^{\boldsymbol{o}}$ frame, is calculated by applying Hooke's law:

$$
\left[\begin{array}{c}
\sigma_{11} \\
\sigma_{22} \\
\sigma_{33} \\
\sigma_{23} \\
\sigma_{13} \\
\sigma_{12}
\end{array}\right]=\boldsymbol{C}_{\mathrm{ij}}\left[\begin{array}{c}
\varepsilon_{11} \\
\varepsilon_{22} \\
\varepsilon_{33} \\
2 \varepsilon_{23} \\
2 \varepsilon_{13} \\
2 \varepsilon_{12}
\end{array}\right]
$$

Finally, by writing the stress tensor $\sigma_{a^{\circ} b^{\circ} c^{\circ}}$ in the $3 \times 3$ matrix format and applying Eqn (6), the stress tensor $\sigma_{x y z}$ is obtained. Note that this analysis depends on unambiguous knowledge of relaxed crystal lattice parameters and would not be possible for minerals such as plagioclase feldspar with compositional substitutions. Quartz is ideal in this respect because substitutions are minimal and deviations from trigonal symmetry can be used to evaluate residual lattice strain. In the figures below and in the paper we will only show the strain components along the main sample axes $\varepsilon_{\mathrm{xx}}, \varepsilon_{\mathrm{yy}}$ and $\varepsilon_{\mathrm{zz}}$.

\section{Section 3. Details of scans}

\subsection{Synthetic quartz crystal}

In the main paper we mentioned that a synthetic quartz crystal was used to calibrate the experiment and here we give some details. A $100 \mu \mathrm{m} \times 100 \mu \mathrm{m}$ area was scanned with 2 $\mu \mathrm{m}$ scanning step size on the thin section of a synthetic quartz single crystal to confirm the angular and strain resolution of the $\mu$ XRD technique. The local orientation is defined by Euler angles with Bunge convention $\phi_{1} \Phi \phi_{2}$ (SM Figure 1a). We observe that angle $\Phi$ is very low. This indicates that the quartz $c$-axis is almost perpendicular to the section. There a regular variation in the range of $0.02^{\circ}$ which is indicative of some bending, probably caused by sample preparation. At such low $\Phi$ angles there is a strong correlation between angles $\phi_{1}$ and $\phi_{2}$. For $\Phi$ minimal noise is observed, even with a tiny angular step of $0.005^{\circ}$, confirming that the orientation resolution of the $\mu$ XRD technique is better than $0.01^{\circ}$. From the strain distribution maps we observe a regular pattern of lattice strain of about $\pm 1.5 \times 10^{-4}$, compressional along $\varepsilon_{\mathrm{yy}}$ and extensional along $\varepsilon_{\mathrm{zz}}$ (SM- Figure $1 b$ ), which is related to the crystal bending expressed in the orientation maps 
(SM- Figure 1a). Such a strain corresponds to a stress of about $15 \mathrm{MPa}$ (see main paper). The scatter in the background pattern, e.g. green and yellow in SM-Figure 1a for $\varepsilon_{\mathrm{xx}}$ is $0.5 \times 10^{-4}$ and represents the resolution for residual strain, corresponding to $5 \mathrm{MPa}$ in stress.

(a)
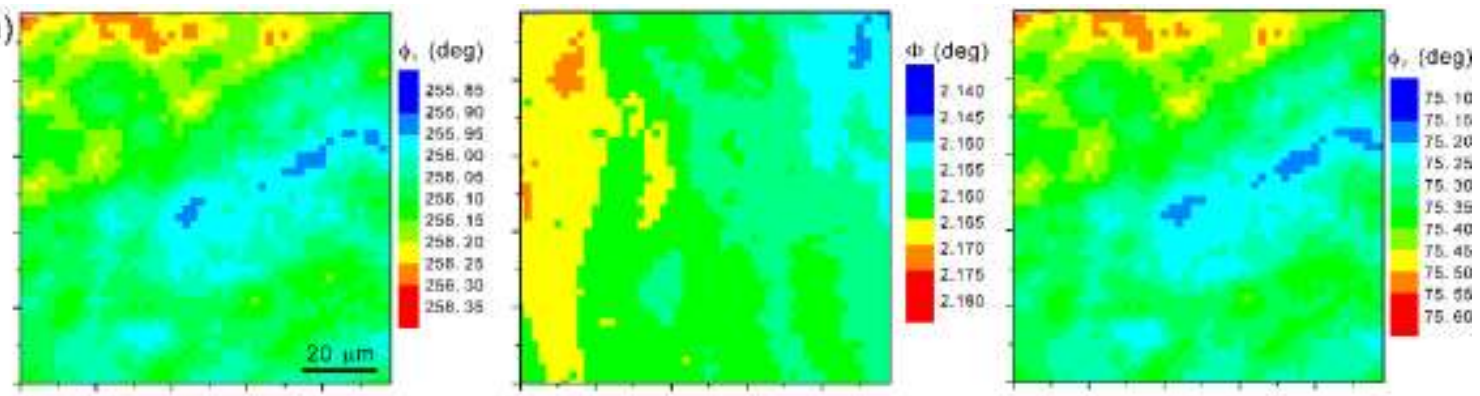

(b)
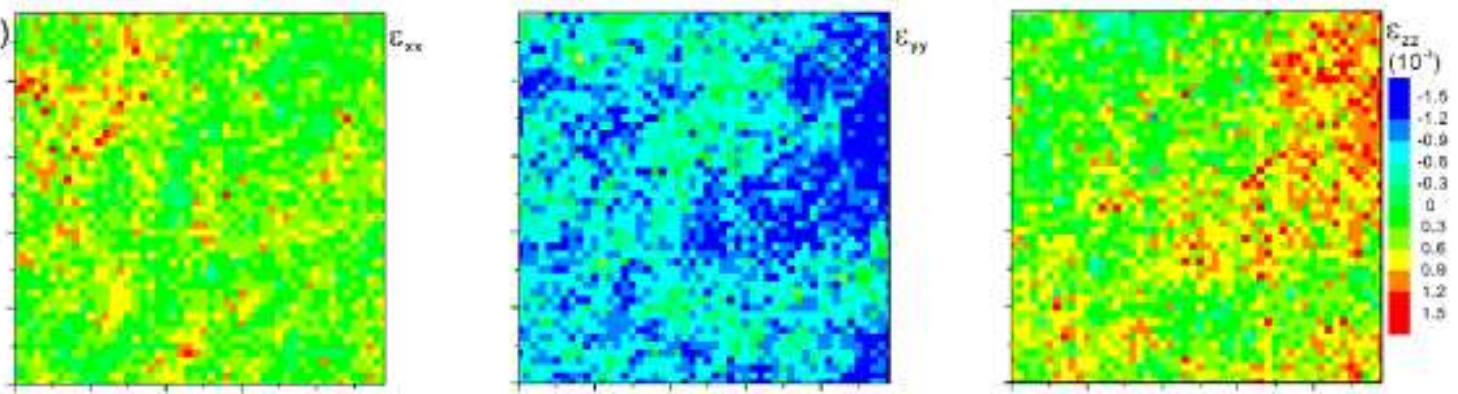

SM-Figure 1 Scan of thin section of a synthetic quartz crystal. (a) Orientation maps $\left(\phi_{1}\right.$, $\left.\Phi, \phi_{2}\right)$, (b) strain component maps.

\subsection{Four scans of SAFOD quartz}

Four scans were conducted on 3 quartz crystals from the SAFOD cataclasite and optical microscope images are shown in SM-Figure 2. Scanned areas are indicated by red rectangles. Scan 1 (SM-Fig. 2a) is discussed in detail in the main body of the paper. The scanning step size is $4 \mu \mathrm{m}$ for all scans, and the scanned area from Scan 1 to Scan 4 is $320 \times 560 \mu \mathrm{m}^{2}, 400 \times 300 \mu \mathrm{m}^{2}, 400 \times 600 \mu \mathrm{m}^{2}$, and $320 \times 240 \mu \mathrm{m}^{2}$, respectively. For all scans Euler angle maps as well as strain distribution maps for the main sample axes are shown (SS-Figures 3-6). For $\phi_{1}$ we used a full color ramge of $30^{\circ}$, for $\Phi$ a range of $10^{\circ}$ to highlight the misorientations within the quartz grains. For $\phi_{2}$ we used a full color range of $120^{\circ}$ to explore Dauphiné twinning; $\phi_{2}$ is the angle of rotation around the crystal $c$-axis and $60^{\circ}$ is the rotation between twins. Note that all the strain components are now in the range of $-1.5 \times 10^{-3}$ to $+1.5 \times 10^{-3}$, i.e. more than an order of magnitude higher than they were for the synthetic quartz. 


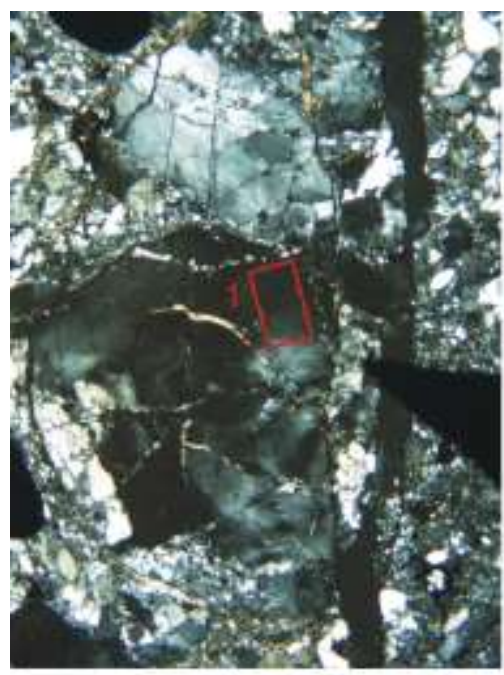

(a)

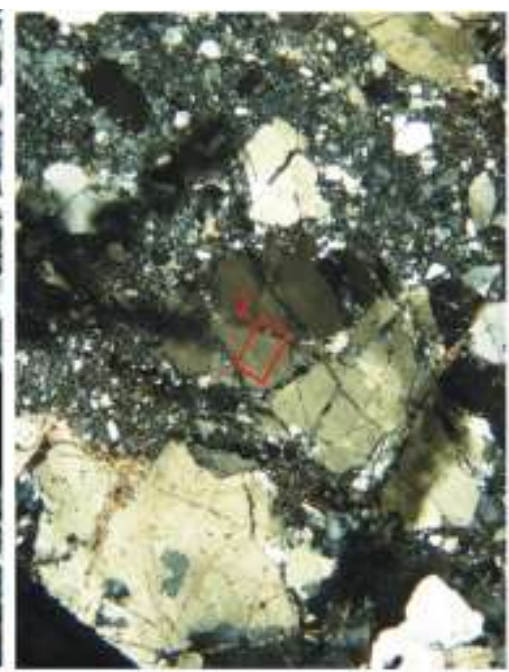

(b)

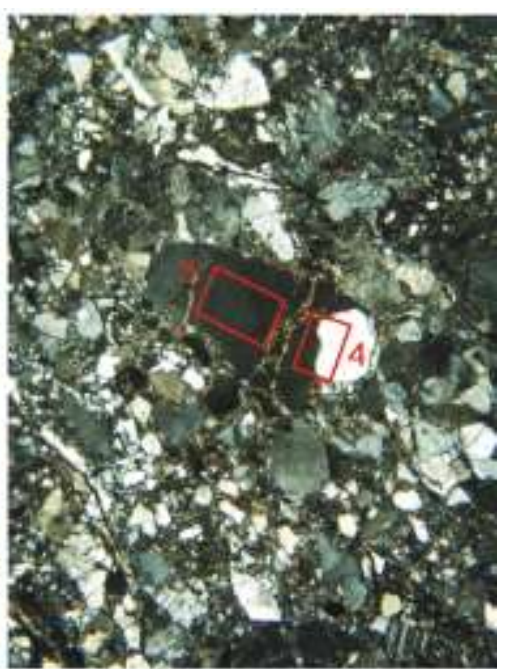

(c)

SM-Figure 2. Petrographic thin section images of SAFOD cataclasite indicating 4 scanned areas on fragmented quartz grains. Crossed polars.

In Scan 1 (SM-Figures 3), we observe orientation changes of $10^{\circ}$ in the $\Phi$ map. Also, there are many small domains $(<10 \mu \mathrm{m})$ with distinct misorientations. Some Dauphiné twins are visible in the $\phi_{2}$ map, mostly yellow-brown regions at the top and near the center of the scan. The strain is extensional along the horizontal (positive $\varepsilon_{\mathrm{xx}}$ ) direction, and compressional in the vertical direction (negative $\varepsilon_{\mathrm{yy}}$ ). The strain component perpendicular to the thin section specimen surface, $\varepsilon_{\mathrm{zz}}$, is close to zero, which is also true for most of the area in the other three scans. It may be related to stress release perpendicular to the thin section surface. Strain concentrations are in small domains, in some cases correlating with misorientation domains. This is the scan discussed in detail in the paper. 
(a)

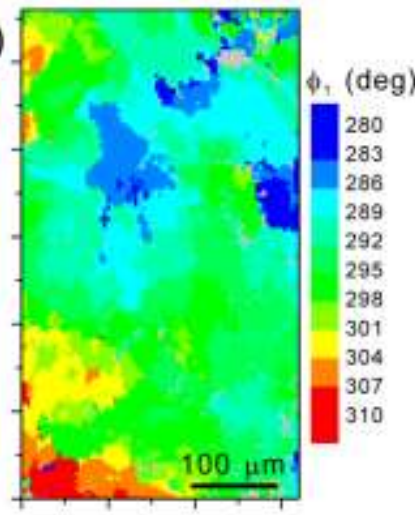

(b)

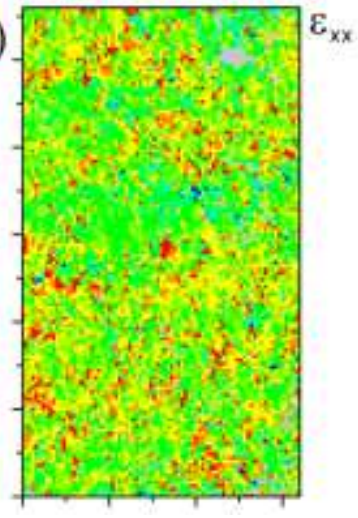

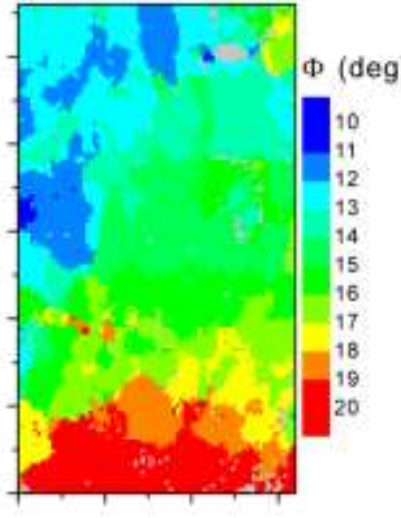

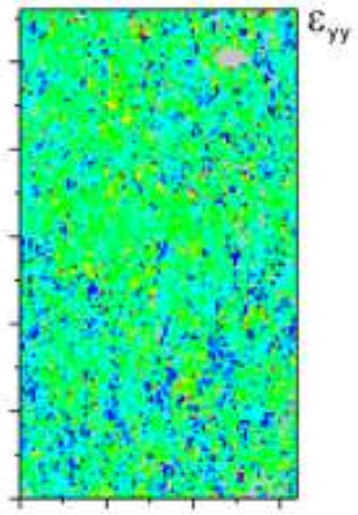

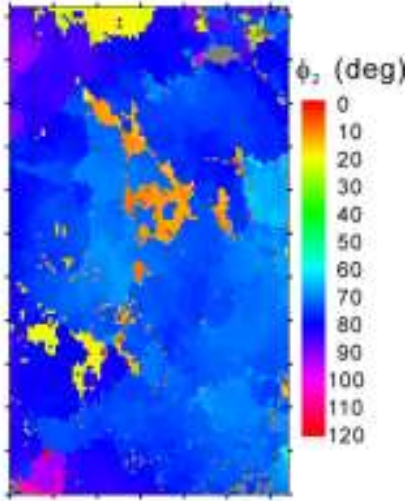

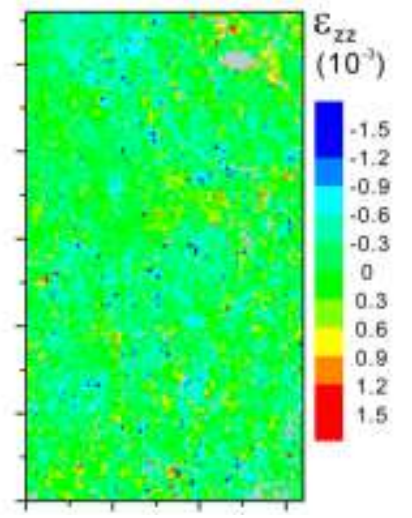

SM-Figure 3. Scan 1 of thin section of a quartz crystal in SAFOD cataclasite. This is the scan discussed in detail in the main paper. For location on the thin section see SM-Figure 2a. (a) Orientation maps $\left(\phi_{1}, \Phi, \phi_{2}\right)$, (b) strain component maps.

In Scan 2 (SM-Figure 4), a subgrain boundary is clearly demonstrated in the $\phi_{1}$ and $\Phi$ map, between the yellow and blue/green regions. Some of the spots along the boundary (red and blue colors) may be due to misindexing. The subgrain boundary is hardly seen in the strain maps. The strain is compressional along the $\boldsymbol{x}$-axis and extensional along the $\boldsymbol{y}$ axis, again with concentrations in distinct domains.

(a)

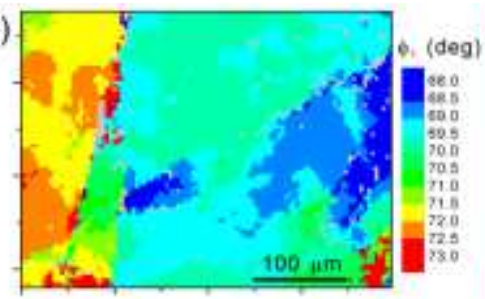

(b)

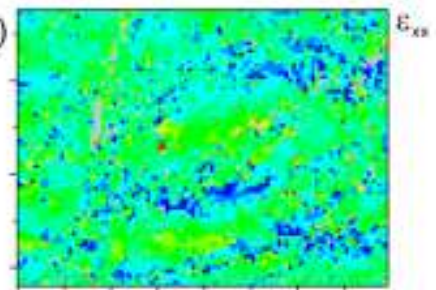

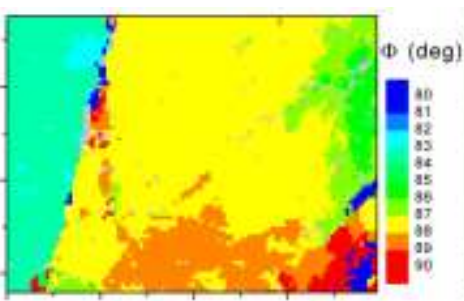

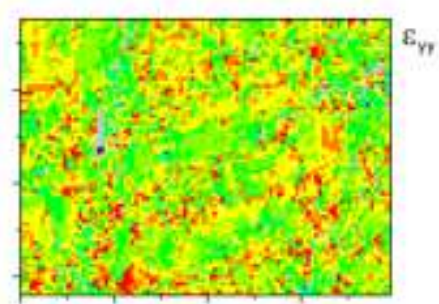

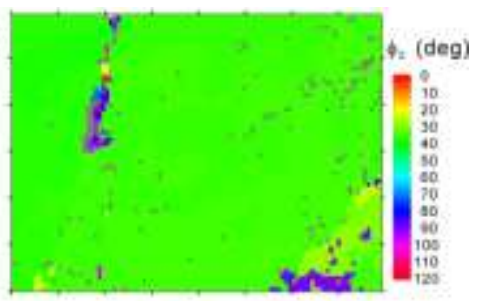

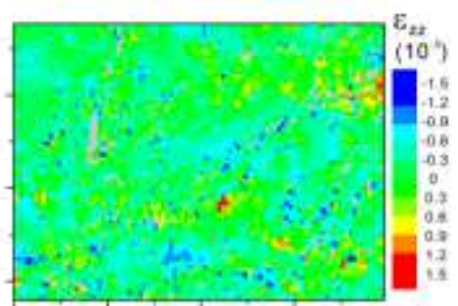

SM-Figure 4. Scan 2 of thin section of quartz crystal in SAFOD cataclasite (SM-Figure $2 a)$. (a) Orientation maps $\left(\phi_{1}, \Phi, \phi_{2}\right)$, (b) strain component maps. 
In Scan 3 (SM-Figure 5), an orientation gradient of the crystal $\boldsymbol{c}$-axis is clearly seen in the $\phi_{1}$ and $\Phi$ maps, i.e. the angle decreases almost continuously from top to bottom, about $10^{\circ}$ in $\phi_{1}$ and $8^{\circ}$ in $\Phi$. In the $\phi_{2}$ map the blue area in the upper left corner is a Dauphiné twin. The orientation gradient may have been generated by plastic deformation preceding the shock deformation. In the strain maps the most distinct features are compressional regions for $\varepsilon_{\mathrm{xx}}$ and extensional regions $\varepsilon_{\mathrm{zz}}$ for in the upper part of the scan. Whether or not this is correlated with the observed orientation gradient would require careful analysis of individual peak shape gradients. It appears that some of the strain concentrations are associated with Dauphiné twins.

(a)

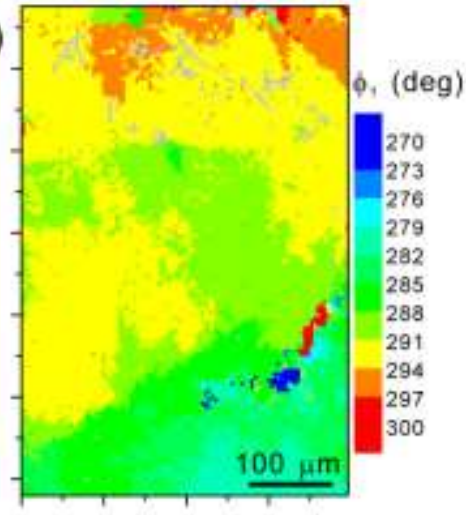

(b)

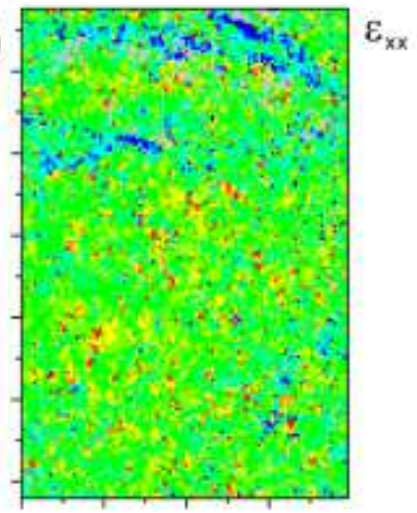

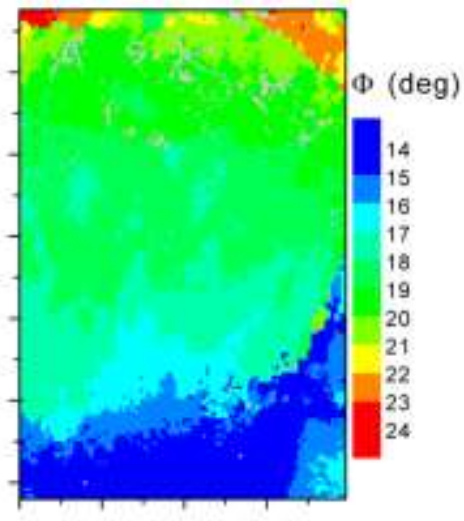

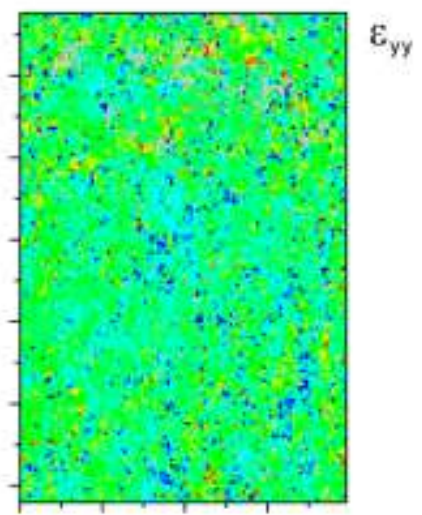

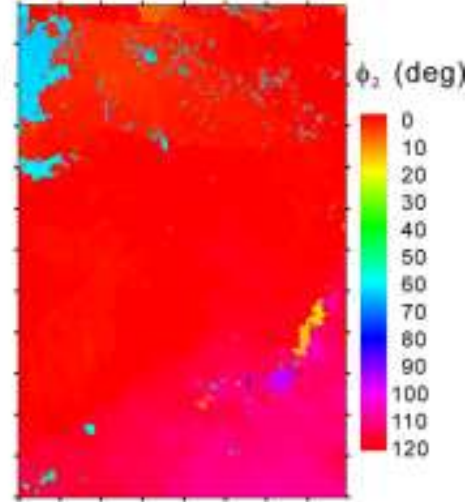

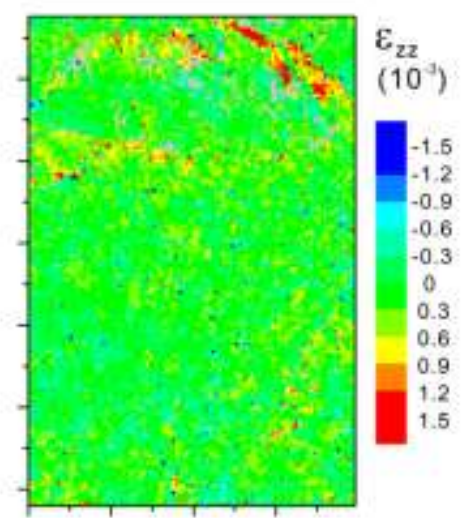

SM-Figure 5. Scan 3 of thin section of quartz crystal in SAFOD cataclasite (SM-Figure $2 b)$. (a) Orientation maps $\left(\phi_{1}, \Phi, \phi_{2}\right)$, (b) strain component maps.

Scan 4 (SM-Figure 6) was taken on an optically fairly homogeneous grain without visible fragmentation (SM-Figure 2c). There is again bending, best expressed in the $\phi_{1}$ map, and also here with microdomains. Also, there are two grains, one best visible with blue colors for $\Phi$ at the top. The $\phi_{2}$ map shows Dauphiné twinned areas (green). Strain maps are more heterogeneous with high values in small domains. 
(a)

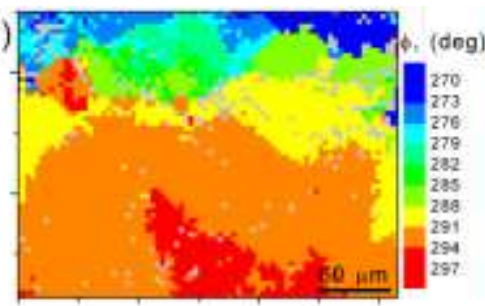

(b)

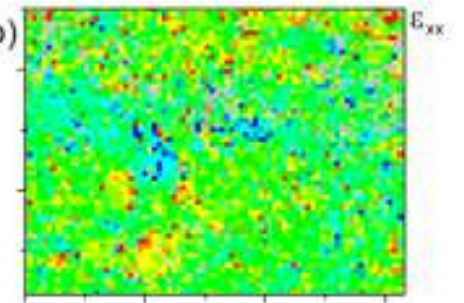

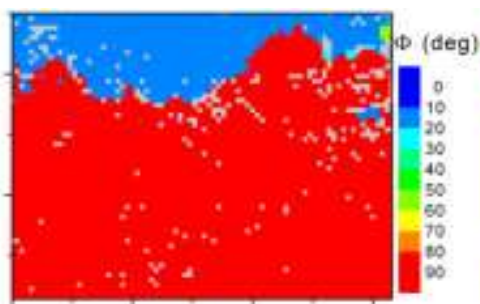
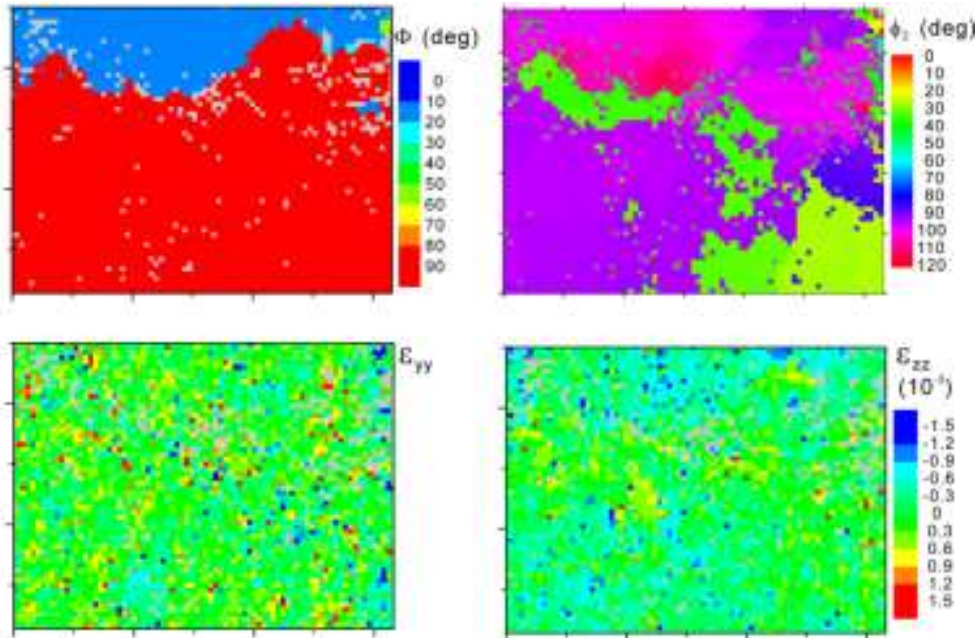

SM-Figure 6. Scan 4 of thin section of quartz crystal in SAFOD cataclasite (SM-Figure $2 c)$. (a) Orientation maps ( $\left.\phi_{1}, \Phi, \phi_{2}\right)$, (b) strain component maps.

In three scans we could document regions with Dauphiné twins. These features could be related to shock deformation events. Mechanical twins have originally been described by Zinserling and Schubnikow (1933) and were observed in quartz fragments from pseudotachylite veins and quartz subjected to meteorite impact (Wenk et al., 2011). In combination with residual stress analyses this could be the subject of a future study.

\section{References:}

Matthies, S., Wenk, H.R., and Vinel, G.W., 1988, Some basic concepts of texture analysis and comparison of three methods to calculate orientation distributions from pole figures: Journal of Applied Crystallography, v. 21, p. 285-304, doi: 10.1107/s0021889888000275.

Pavese, A., 2005, About the relations between finite strain in non-cubic crystals and the related phenomenological P-V Equation of State: Physics and Chemistry of Minerals, v. 32, p. 269-276, doi: 10.1007/s00269-005-0465-8.

Sih, G.C., and Tang, X.S., 2006, Simultaneous occurrence of double micro/macro stress singularities for multiscale crack model: Theoretical and Applied Fracture Mechanics, v. 46, p. 87-104, doi: 10.1016/j.tafmec.2006.07.001.

Wawszczak, R., Baczmański, A., Braham, C., Seiler, W., Wróbel, M., Wierzbanowski, K., and Lodini, A., 2011, Residual stress field in steel samples during plastic deformation and recovery processes: Philosophical Magazine, v. 91, p. 2263-2290, doi: 10.1080/14786435.2011.557401.

Wenk, H.-R., Janssen, C., Kenkmann, T. and Dresen, G., 2011, Mechanical twinning in quartz: shock experiments, impact, pseudotachylites and fault breccias: Tectonophysics, v. 510, p. 69-79.

Withers, P.J., and Bhadeshia, H.K.D.H., 2001, Residual stress. Part 2 - Nature and origins: Materials Science and Technology, v. 17, p. 366-375, doi: 10.1179/026708301101510087.

Zinserling, K., and Schubnikov, A., 1933, Ueber die Plastizitaet des Quarzes: Zeitschrift fuer Kristallographie, v. 85, p. 454-461. 